\title{
Importância da ultra-sonografia ocular na avaliação pré-operatória de pacientes com catarata total
}

\author{
The importance of ocular ultrasonography in the preoperative evaluation of patients \\ with maturecataract
}

\author{
ÁlvaroPedrosode Carvalho Lupinacci ${ }^{1}$ \\ Rafael Vanini ${ }^{1}$ \\ David Leonardo Cruvinel Isaac ${ }^{2}$ \\ Vinícius Coral Ghanem² $^{2}$ \\ Carlos Eduardo Leite Arieta ${ }^{3}$
}

Trabalho realizado no Departamento de Oftalmologia/ Otorrinologia da Universidade Estadual de Campinas - UNICAMP.

${ }^{1}$ Acadêmicos do $6^{\circ}$ ano de medicina da Faculdade de Ciências Médicas da Universidade Estadual de Campinas UNICAMP

${ }^{2}$ Médicos residentes do $3^{\circ}$ ano em Oftalmologia do Hospital de Clínicas da Universidade Estadual de Campinas UNICAMP.

${ }^{3}$ Professor assistente doutor do Departamento de Oftalmologia do Hospital de Clínicas da Universidade Estadual de Campinas - UNICAMP.

Endereço para Correspondência: Álvaro Pedroso de Carvalho Lupinacci, Rua Frei José de Monte Carmelo, 463 - Campinas (SP) CEP 13026-461

E-mail: lupinacci@mpcnet.com.br

Recebido para publicação em 07.11.2002

Versão revisada recebida em 29.04.2003

Aprovação em 27.06.2003

Nota Editorial: Pela análise deste trabalho e por sua anuência na divulgação desta nota, agradecemos ao Dr. Ayrton Roberto Branco Ramos.

\begin{tabular}{|l|}
\hline RESUMO \\
\hline Com os avanços da cirurgia para catarata, o prognóstico da recuperação da \\
visão aumentou drasticamente, tornando-se necessário prever resultados \\
indesejados para a acuidade visual no pós-operatório. Objetivos: Verificar \\
a importância da ultra-sonografia ocular na avaliação pré-operatória do \\
segmento posterior em pacientes com catarata madura, e relacionar possí- \\
veis doenças que possam comprometer os resultados cirúrgicos, sua \\
prevalência e seus principais fatores de risco. Métodos: Foi realizadoestudo \\
retrospectivo do prontuário de 262 pacientes em que houve a necessidade \\
de ultra-sonografia ocular devido à presença de catarata madura, impedindo \\
assima avaliaçãofundoscópica do segmento posterior. Também foi avaliada \\
a relação entre a presença dessas alterações e o sexo, idade, raça, história de \\
trauma ocular, doenças sistêmicas e oculares e presença de catarata no olho \\
contralateral. Resultados:Encontraram-se alterações ecográficas em $24,8 \%$ \\
dos exames realizados entre 1996 e 2001, dos quais odescolamento de retina \\
(9,9\%)e as condensações vítreas(9,9\%) foram as mais comuns. Conclusões: \\
Dentre os fatores de risco levantados como predisponentes para achados \\
de ultra-sonografia ocular, a uveíte foi o único elemento estatisticamente \\
significante. Não foi possível, pelos dados obtidos, correlacionar positiva- \\
mente o trauma ocu-lar às patologias de segmento posterior descritas. \\
\hline
\end{tabular}

Descritores: Catarata/ultra-sonografia; Descolamento retiniano; Fatores de tempo; Cuidados pré-operatórios; Fundo de olho; Oftalmoscopia/métodos

\section{INTRODUÇÃO}

Com o avanço da cirurgia de catarata e aumento da exigência dos pacientes por melhores resultados visuais e do oftalmologista por uma maior previsibilidade destes, é de fundamental importância determinar a existência de outras alterações oculares e, quando possível, seus efeitos visuais nos pacientes portadores de catarata. Para isto, são utilizados atualmente, testes para avaliação do potencial visual desses pacientes, dentre estes o medidor da acuidade potencial (potencial acuity meter - PAM) e a interferometria ${ }^{(1-3)}$ são os mais utilizados. Estes exames, porém, não apresentam boa confiabilidade nos casos de pacientes com catarata madura e acuidade visual muito baixa ${ }^{(2)}$. Nestes casos a ultra-sonografia, apesar de não determinar a acuidade visual potencial, é o exame mais utilizado para se diagnosticar eventuais alterações anatômicas no segmento posterior que possam comprometer o resultado visual pós-operatório ${ }^{(1,4-6)}$.

O objetivo deste estudo é verificar a importância da ultra-sonografia ocular na avaliação pré-operatória de pacientes com catarata madura, verificando a prevalência dos achados ecográficos e seus possíveis fatores de risco. 


\section{MÉTODOS}

Realizou-se análise retrospectiva do prontuário de 262 pacientes consecutivos (262 olhos), do Hospital das Clínicas da Universidade Estadual de Campinas (HC-Unicamp), que foram submetidos à ultra-sonografia ocular como avaliação pré-operatória para cirurgia de catarata total, entre os anos de 1996 e 2001. A ecografia ocular foi realizada quando não era possível a avaliação fundoscópica adequada. Utilizaram-se como critérios de exclusão: pacientes com idade menor que 18 anos, uma vez que se pretendia avaliar a catarata senil, história de trauma perfurante ou cirurgia ocular prévia e doenças previamente conhecidas do segmento posterior ${ }^{(1)}$.

A ecografia $B$ diagnóstica foi realizada por residentes do terceiro ano de oftalmologia, sob a orientação de médicos assistentes do setor de retina e vítreo, utilizando um ecógrafo Allergan Humphrey A/B scan system 835.

De acordo com os achados ecográficos dividiu-se os pacientes em 2 grupos: Normal (pacientes com descolamento do vítreo posterior [DVP] foram incluídos neste grupo) e Alterado (pacientes que apresentavam alguma alteração ecográfica, excetuando-se descolamento do vítreo posterior).

Para determinação dos possíveis fatores de risco de ocorrência de alterações ecográficas avaliou-se: idade, sexo, raça (classificou-se em brancos e não-brancos, devido à baixa freqüência de negros e amarelos), doenças sistêmicas (diabetes, hipertensão ou outras), trauma não perfurante, doenças oculares (uveíte, exotropia ou outras) e grau evolutivo da catarata no olho contralateral. Quanto aos graus evolutivos classificou-se em 4 grupos: a) Leve, que possibilitava fácil avaliação fundoscópica; b) Moderado, na qual a avaliação fundoscópica era possível, mas prejudicada pela opacidade; c) Madura, quando o exame fundoscópico era impossível; d) Operado, agrupando pacientes previamente submetidos à cirurgia de catarata em um dos olhos.

\section{ANÁLISE ESTATÍSTICA}

Para a comparação de variáveis contínuas entre os dois grupos foi utilizado o teste de Mann-Whitney. Para comparar proporções ou verificar associação, foi utilizado o teste Qui-quadrado ou o teste Exato de Fisher, quando necessário ${ }^{(7-8)}$ e na identificação de fatores associados aos achados ecográficos observados utilizou-se a análise de regressão logística ${ }^{(9)}$ - modelo logito sendo o nível de significância adotado de $5 \%$, ou p $\leq 0,05$.

Utilizou-se, para a análise dos dados obtidos, o programa de computador SAS System for Windows (Statistical Analysis System), versão 8.1 elaborado pela SAS Institute Inc.

\section{RESULTADOS}

Dos 262 pacientes avaliados, $146(55,7 \%)$ eram do sexo masculino e $116(44,3 \%)$ do sexo feminino. A idade média foi de 58,6 anos $( \pm 17,5)$, variando entre 18 e 107 anos, sendo $220(84 \%)$ pacientes da raça branca e 42 (16\%) não brancos. Observou-se que 79 pacientes $(30,2 \%)$ eram portadores de hipertensão arterial sistêmica (HAS), $31(11,8 \%)$ de diabetes e $56(21,4 \%)$ de outras doenças sistêmicas, como: cardiopatia, nefropatia, mieloma múltiplo ou SIDA. As alterações oculares encontradas, através do exame oftalmológico, concomitantes à presença de catarata foram: exotropia em $16(6,1 \%)$ pacientes, uveíte em $35(13,4 \%)$ e outras doenças oculares como: leucoma, glaucoma e tumor conjuntival em 21 (8\%). História prévia de trauma ocular contuso, de intensidade e natureza variadas, foi encontrada em 52 $(19,9 \%)$ pacientes. Na tabela 1, encontram-se as variáveis previamente descritas distribuídas nos dois grupos estudados (Normal e Alterado). Ao se analisar o olho contralateral, 144 (55\%) pacientes apresentaram catarata de grau Leve, $54(20,6 \%)$ Moderado, 29 (11\%) Madura e $35(13,4 \%)$ Operados.

Dentre os pacientes que apresentavam alterações ecográficas (exceto DVP), observamos condensações vítreas em 26

\begin{tabular}{|c|c|c|c|c|c|c|}
\hline \multirow[b]{2}{*}{ Idade } & \multicolumn{2}{|c|}{ Grupo } & \multirow{2}{*}{$\begin{array}{c}\text { normal } \\
17,5\end{array}$} & \multicolumn{2}{|c|}{ Grupo alterado } & \multirow{2}{*}{$\begin{array}{l}\text { Coeficiente de significância }(p) \\
\qquad 0,0381^{*}\end{array}$} \\
\hline & 59 & $\mathrm{Dp}$ & & $52,6 \mathrm{Dp}$ & 20 & \\
\hline \multirow[t]{2}{*}{ Sexo } & M & 109 & $(55,3 \%)$ & M 37 & $(57 \%)$ & 0,823 \\
\hline & $\mathrm{F}$ & 88 & $(44,7 \%)$ & $\mathrm{F} 2 \varepsilon$ & $(43 \%)$ & \\
\hline \multirow[t]{2}{*}{ Raça } & B & 168 & $(85,3 \%)$ & B 52 & $(80 \%)$ & 0,314 \\
\hline & NB & 29 & $(14,7 \%)$ & NB 13 & $(20 \%)$ & \\
\hline Hipertensão arterial sistêmica & & 66 & $(33,5 \%)$ & 13 & $(20 \%)$ & 0,0397 \\
\hline Diabetes mellitus & & 26 & $(13,2 \%)$ & 5 & $(7,7 \%)$ & 0,233 \\
\hline Outras doenças sistêmicas & & 46 & $(23,4 \%)$ & 10 & $(15,4 \%)$ & 0,174 \\
\hline Exotropia & & 12 & $(6,1 \%)$ & & $(6,2 \%)$ & 1,000 \\
\hline Uveíte & & 20 & $(10,2 \%)$ & 15 & $(23,1 \%)$ & 0,008 \\
\hline Outras doenças oculares & & 17 & $(8,6 \%)$ & & $(6,2 \%)$ & 0,524 \\
\hline Trauma & & 37 & $(18,8 \%)$ & 1 & $(23,1 \%)$ & 0,463 \\
\hline Total & & 197 & $(75,2 \%)$ & 6 & $(24,8 \%)$ & \\
\hline
\end{tabular}


casos $(9,9 \%)$. O descolamento de retina total ou parcial foi evidenciado em 26 casos $(9,9 \%)$, dos quais, 18 (6,9\%) havia descolamento total da retina e em 8 casos $(3,1 \%)$, descolamento parcial. Neste grupo foram reunidos tanto DR regmatogênico, tracional e misto. As demais alterações encontradas são descritas na tabela 2.

Através do teste do Qui-quadrado, na análise univariada, observou-se uma relação positiva entre o encontro de alterações ecográficas e hipertensão arterial $(\mathrm{p}=0,04)$ ou uveíte $(\mathrm{p}=0,008)$.

Pela regressão logística univariada, para a presença de achados ecográficos, obteve-se um valor de $p$ para a variável idade de 0,0151, para presença hipertensão de 0,0422 e para história positiva de uveíte de 0,0096. As demais variáveis estudadas apresentaram um valor de $p$ maior que o nível de significância de 0,05 (Tabela 3). Assim, constatou-se que a presença de uveíte relaciona-se, de maneira estatisticamente significativa, ao encontro de alterações ecográficas em um maior número de pacientes. Ao se testar as interações estatísticas entre idade, hipertensão e uveíte, não se observou nível de significância de 5\%. No modelo multivariado, somente a presença de uveíte foi significativa ao nível de 5\%. Não foi observada relação estatisticamente significativa entre o trauma ocular e a presença ou ausência de alterações ecográficas.

\section{DISCUSSÃO}

Diversas alterações encontradas na ecografia B de pacientes com catarata madura podem interferir no prognóstico visual ou até mesmo contra-indicar a cirurgia de catarata. Alterações ecográficas no segmento posterior foram descritos na literatura ${ }^{(1)} \mathrm{em} \mathrm{19,6 \%}$ dos pacientes com catarata densa. No presente estudo $24,8 \%$ dos pacientes examinados apresentavam alterações ecográficas, demonstrando a importância da realização deste exame como avaliação pré-operatória em pacientes portadores de catarata densa. O descolamento de retina (DR) foi a doença ocular mais observada, sendo diagnosticada em 26 casos $(9,9 \%)$, em concordância com a literatura revisada ${ }^{(10)}$, que descreveu DR em 26 casos $(9,7 \%)$ de 269 analisados. O diagnóstico do DR através da ultra-sonografia ocular permite a avaliação de suas características, permitindo a conduta adequada para cada caso.

\begin{tabular}{|lccc|}
\hline \multicolumn{4}{c}{ Tabela 2. Alterações ecográficos pré-operatórios, de pacientes com catarata total } \\
Achados ecográficos & No de pacientes & Porcentagem do total & Porcentagem dos alterados \\
Condensações vítreas & 26 & 9,9 & 40 \\
Descolamento de retina total & 18 & 6,9 & 27,7 \\
Descolamento de retina parcial & 8 & 3,1 & 12,3 \\
Phthisis bulbi & 6 & 2,3 & 9,2 \\
Escavação aumentada & 2 & 0,8 & 3,1 \\
Estafiloma de polo posterior & 2 & 0,8 & 3,1 \\
Coloboma de nervo óptico & 1 & 0,4 & 1,5 \\
Drusas de papila & 1 & 0,4 & 1,5 \\
Tumor & 1 & 0,4 & 1,5 \\
Total & 65 & $\mathbf{2 4 , 8}$ & $\mathbf{1 0 0}$ \\
& & & \\
\end{tabular}

\begin{tabular}{|c|c|c|c|c|}
\hline Variável & Grupo comparado & p-valor & Odds ratio & IC $95 \%$ \\
\hline Idade & Idade & 0,0151 & 0,981 & 0,$967 ; 0,996$ \\
\hline Sexo & $M \times F$ & 0,8226 & 1,067 & 0,$606 ; 1,878$ \\
\hline Raça & $N B \times B$ & 0,3161 & 1,448 & 0,$702 ; 2,989$ \\
\hline DM & Sim $x$ Não & 0,2391 & 0,548 & 0,$201 ; 1,492$ \\
\hline HAS & Sim $\times$ Não & 0,0422 & 0,496 & 0,$252 ; 0,975$ \\
\hline Outras DS & Sim x Não & 0,1776 & 0,597 & 0,$282 ; 1,264$ \\
\hline Uveíte & Sim x Não & 0,0096 & 2,655 & 1,$268 ; 5,561$ \\
\hline Exotropia & Sim x Não & 0,9854 & 1,011 & 0,$314 ; 3,251$ \\
\hline Outras DO & Sim x Não & 0,5261 & 0,694 & 0,$225 ; 2,144$ \\
\hline Trauma & Sim $\times$ Não & 0,4633 & 1,289 & 0,$654 ; 2,542$ \\
\hline CAT-C1 & Moderada x Leve & 0,1119 & 0,534 & 0,$246 ; 1,157$ \\
\hline CAT-C2 & Madura $\times$ Leve & 0,5360 & 0,747 & 0,$297 ; 1,880$ \\
\hline CAT-C3 & Operada $\times$ Leve & 0,0693 & 0,391 & 0,$142 ; 1,077$ \\
\hline
\end{tabular}


Condensações vítreas também foram observadas em 26 pacientes $(9,9 \%)$, e na maior parte dos casos o seu encontro não constituiu fator de contra-indicação cirúrgica.

Não foi observada relação estatisticamente significativa entre o trauma ocular e alterações de segmento posterior. Mesmo se esperando uma maior incidência de DR nos pacientes com história de trauma contuso ocular, como relatado em artigo revisado $^{(1)}$, essa relação não foi observada no presente estudo, por dificuldade de verificar se o trauma referido era responsável pela catarata (catarata traumática), ou se havia catarata senil, e o paciente relacionava a perda visual gradual a um eventual trauma ocular, mesmo que corriqueiro. Porém, em outra publicação ${ }^{(10)}$, foi descrito $9,7 \%$ de DR em pacientes sem história de trauma ocular e $38,1 \%$ naqueles que apresentavam esse antecedente em sua história, sendo que esta diferença se mostrou estatisticamente significativa $(\mathrm{p}=0,001)$.

\section{CONCLUSÃO}

Assim, através dos resultados obtidos neste estudo, referentes às alterações ecográficas encontradas em pacientes portadores de catarata madura, e dos dados disponíveis na literatura, confirmou-se que a ultra-sonografia ocular constitui importante exame pré-operatório a ser realizado em pacientes cuja catarata impeça ou dificulte a avaliação adequada das estruturas do segmento posterior do globo ocular, sendo um eficiente método de avaliação, permitindo o diagnóstico de doenças do pólo posterior que podem alterar a indicação cirúrgica.

\section{ABSTRACT}

With the new techniques of cataract surgery visual success has increased and it became necessary to foresee undesirable postoperative results regarding visual acuity. Purpose: To verify the role of ocular ultrasonography in the preoperative evaluation of patients with mature cataract searching for pos- terior segment pathologies that may affect surgical results, their prevalence and the main associated risk factors. Methods: A retrospective study was performed analyzing records of 262 patients indicated for ocular ultrasonography because the presence of mature cataract, thus preventing fundoscopic evaluation of the posterior segment. It was also tried to associate the presence of those changes with sex, age, race, history of ocular trauma, systemic and ocular diseases and the presence of cataract in the contralateral eye. Results: Ecographic changes were found in $24.8 \%$ of the examinations performed between 1996 and 2001, of which retinal detachment and vitreous condensations were the most common, with $9.9 \%$ of the findings for each. Conclusions: Among the risk factors pointed out as predisposing to findings in ocular ultrasonography, uveitis was the only statistically significant element. It was not possible to correlate positively the described posterior segment pathology to ocular trauma using obtained data.

Keywords: Cataract/ultrasonography; Retinal detachment; Time factors; Preoperative care; Fundus oculi; Ophthalmoscopy

\section{REFERÊNCIAS}

1. Anteb Y II, Blumenthal EZ, Zamir E, Waindim P. The role of preoperative ultrasonography for patients with dense cataract: a retrospective study of 509 cases. Ophthalmic Surg Lasers 1998;29:114-8.

2. Datiles MB, Lasa MS, Freidlin V. A longitudinal study of cortical cataracts using retroillumination photographs. Curr Eye Res 1996;15:53-61.

3. Barret BT, Davison PA, Eustace P. Clinical comparison of three techniques for evaluating visual function behind cataract. Eye 1995;9(Pt6):722-7.

4. Haile M, Mengistu Z. B-scan ultrasonography in ophthalmic diseases. East Afr Med 1996;73:703-7.

5. Hurst MA, Douthwaite WA. Assessing vision behind cataract: a review of methods. Optom Vis Sci 1993;70:903-13.

6. Fielding JA. Imaging the eye with ultrasound. Br J Hosp Med 1992;47:805-15.

7. Conover WJ. Practical nonparametric statistics. New York: John Wiley \& Sons; 1971.

8. Fleis JL. Statistical methods for rates and proportions. 2a ed. New York; John Wiley \& Sons; 1981.

9. Hosmer DW, Lemeshow S. Applied logistic regression. New York: John Wiley \& Sons; 1988.

10. Corrêa ZMS, Goldhardt R, Marcon AS, Marcon IM. Achados ecográficos em pacientes com catarata total. Arq Bras Oftalmol 2002;65:609-13.

\title{
ABO ELETRÔNICO
}

\author{
Novo site
}

Acesso: http://www.abonet.com.br 